\title{
Rechtsgeschichte
}

www.rg.mpg.de

http://www.rg-rechtsgeschichte.de/rg15

Zitiervorschlag: Rechtsgeschichte Rg 15 (2009)

$\operatorname{Rg} 15_{2009}$

$220-222$

http://dx.doi.org/10.12946/rg15/220-222

\section{Gerd Hankel}

Die fragwürdige Verengung des Genozidbegriffs 
men. Christoph Link ruft vergessenes Wissen zur kirchlichen Rechtsgeschichte wieder in Erinnerung und er ergänzt die Betrachtung des heutigen Staatskirchenrechts um eine unverzichtbare his- torische Dimension. Das souverän für zweitausend Jahre geleistet zu haben, ist das große Verdienst dieses Buches.

\section{Die fragwürdige Verengung des Genozidbegriffs*}

Diesem Buch ist zu wünschen, dass es, um eine endgültige Kaufentscheidung treffen zu können, nicht zur Hand genommen und durchgeblättert wird. Denn dann besteht die Gefahr, dass man sich festliest, womöglich weiter hinten im Buch oder gar im durch Kursivierung optisch hervorgehobenen Postskriptum, in dem man auf die vom Autor zustimmend kommentierte Behauptung stößt, dass auch bestimmte Sprachenpolitiken genozidalen Charakter haben können. Bei dieser Behauptung handelt es sich mitnichten um einen Druckfehler oder um eine sinnentstellende Verkürzung: Die Verletzung von Sprachenrechten (language rights) sei eine Form kulturellen Völkermords heißt es beispielsweise an einer Stelle (242), bilinguale Erziehung könne genozidal sein an einer anderen (243). Führt man sich auch nur einen Augenblick vor Augen, wofür der Begriff des Genozids gewöhnlich steht - für den Massenmord an Menschen jeden Alters, denen allein aufgrund ihrer Zugehörigkeit zu einer bestimmten Gruppe die möglichst umfassende Vernichtung zugedacht ist -, wird man große Mühe haben, die Behauptungen des Autors zu akzeptieren und in ihnen nicht nur eine moralisch hanebüchene Banalisierung zu erkennen, die beinahe reflexartig dazu führt, dass das Buch keiner Lektüre für wert befunden und beiseite gelegt wird.
Mit dieser Reaktion wäre allerdings die Chance verpasst, einen Autor kennenzulernen, der, auch wenn man ihm im Ergebnis nicht zustimmt, eine ganze Reihe nachdenkenswerter Gedanken und Einsichten formuliert, die in dieser Prägnanz selten zu lesen sind. Vielleicht liegt dies daran, dass der Autor sein Buch mit einer gehörigen Portion Entrüstung geschrieben hat. Entrüstung darüber, dass sein Heimatland Spanien zigmillionenfaches Unrecht in den früheren lateinamerikanischen Kolonien zu verantworten hat, ein Unrecht, das, wie er schreibt, eigentlich als Genozid hätte bezeichnet werden und, der appellativen Wucht des Begriffs entsprechend, zu Reparationsleistungen hätte verpflichten müssen. Entrüstung aber vor allem darüber, dass der ursprünglich von Raphael Lemkin konzipierte Genozidbegriff von den Staaten als den Hauptakteuren des Völkerrechts auf fatale Weise verkürzt worden ist und dadurch der falsche Blick auf das Kolonialgeschehen wie in Lateinamerika überhaupt erst möglich wurde: Aus einem Verbrechenstypus, der sich ursprünglich nicht nur auf die physische Vernichtung einer Menschengruppe bezog, sondern auch und unabhängig davon deren Auslöschung in kultureller Hinsicht, d. h. in ihren sozialen, politischen, ökonomischen, religiösen usw. Eigenarten umfasste, war ein allein auf den Aspekt des Massenmords

\footnotetext{
* Bartolomé Clavero, Genocide or Ethnocide, I933-2007. How to make, unmake and remake law with words, Mailand: Giuffrè Editore 2008, viii, 268 p., ISBN 978-88-I 4-I 4277-2
} 
begrenztes Verbrechen geworden, das zudem auf der subjektiven Tatseite noch mit dem über den allgemeinen Tatvorsatz hinausgehenden Erfordernis der »Absicht « zur Völkermordbegehung versehen war. Parallel dazu war aus der Rechtsdiskussion noch der Begriff des "Ethnozids" verschwunden, den Lemkin als Alternative zum Genozidbegriff $($ genos $=$ Volk, Rasse; caedere $=$ töten) entwickelt hatte und der durch den Verweis auf den weiteren Begriff der Nation (ethnos) insbesondere auch die nicht-physische Seite der Vernichtung zum Ausdruck bringen sollte. Konvention über die Verhütung und Bestrafung des Völkermords, also des Genozids, war dann aber der Name des am 9. Dezember I948 von der Staatenversammlung angenommenen Übereinkommens, und die darin aufgelisteten Völkermordhandlungen zeigen, so Clavero, deutlich, dass bei der Kodifikation die physische Existenz der geschützten Gruppen im Vordergrund stand. Lediglich unter lit. e werde in Artikel II die Zerstörung einer Gruppenidentität in Form der "gewaltsame[n] Überführung von Kindern der Gruppe in eine andere Gruppe « als Völkermordhandlung genannt.

Doch was unter dem Eindruck der Ermordung der europäischen Juden wenige Jahre zuvor noch verständlich erschien, setzte gleichwohl ein für die Zukunft falsches Zeichen, da es, wie Clavero mit vielen Beispielen belegt, zu einer Verfestigung des reduzierten Genozidverständnisses auf den unter enge rechtliche Tatbestandsmerkmale subsumierbaren Massenmord führte. Und da das Recht sehr viel mit Sprache zu tun hat, spielten unterschiedliche Begrifflichkeiten dabei eine wichtige Rolle. Worte wie »ethnische Säuberung « wurden gebildet, andere wie »Ausrottung " (schon im Nürnberger Statut enthalten, dann Jahrzehnte später in die Statuten der ad hoc-Tribunale und des Internationalen Straf- gerichtshofs übernommen) wurden reaktiviert, wieder andere wie »Ethnozid « oder »kultureller Genozid « von der UN-Generalversammlung zurückgewiesen und nicht in Resolutionen oder Deklarationen, deren Auszüge sich in dem gut 20-seitigen Textanhang des Buchs finden, aufgenommen. Die Folge dieser linguistischen Aktivitäten, ganz zu schweigen von ihren sonstigen Widersprüchen (was z. B. ist der Unterschied zwischen dem Verbrechen der »Ausrottung " als Tatbestandsvariante der Verbrechen gegen die Menschlichkeit und dem nach herrschender Ansicht eng zu fassenden Verbrechen des Genozids?): Die internationale Gemeinschaft nimmt einen Genozid erst dann zur Kenntnis, wenn es zu spät ist, wenn die Massaker begonnen haben. Die Vorstufen, die zum Massenmord führen, werden ignoriert, ihnen fehlt die mobilisierende Wirkung eines Namens, wie sie vom Begriff des Genozids ausgeht.

Diesem Befund Claveros ist, denken wir nur an die lähmende terminologische Auseinandersetzung um das Geschehen in Darfur, wohl uneingeschränkt zuzustimmen. Auch wird man ihm folgen können, wenn er weiter ausführt, dass sich heute indigene bzw. allgemein unter fremder Herrschaft lebende Völker in einer prekären Situation befinden, die in mancher schlimmen Hinsicht dem früheren einheimischen Leben in den Kolonien ähnelt, trotzdem aber weltweit nur geringe Betroffenheit hervorruft. Daran ändert auch die aufrüttelnd gemeinte Bildung von Wörtern wie »Demozid «, »Klassizid «, »Autogenozid « oder »Politizid «, um nur einige wenige dieser »-zid-Neologismen « zu nennen, nichts, weil sie keinerlei rechtliche Wirkung entfalten und nur im sozialwissenschaftlichen Bereich verbleiben. Nein, der Dissens beginnt dort, wo Clavero die Lösung dieses Missstands in einer inhaltlichen Ausweitung des Genozidbegriffs 
oder dessen Ersetzung durch den weiteren Begriff des Ethnozids erblickt. Kulturelle Dominanz, Verdrängung oder Auslöschung als Völkermord oder Vorstufe dazurechnen zu wollen, wird mitnichten einen erhöhten Handlungsdruck internationaler Instanzen bewirken. Ganz im Gegenteil ist zu befürchten, dass dann, wenn beispielsweise sogar massive sprachpolitische Pressionen als genozidale Akte angesehen werden, der Bekämpfung von Genozidverbrechen ein schlechter Dienst erwiesen wird. Wer zu oft und in großer Diskrepanz zum historischen Vorbild der Shoa (angebliche) Genozidverbrechen anprangert, fördert letztlich nur weltweite Indifferenz. Dabei hat Clavero in gewisser Weise durchaus Recht, wenn er die herausgehobene Stellung des Genozidverbrechens kritisiert. Nur

\section{Ringparabeln*}

Seit Mitte der Sechzigerjahre gab es vielerorts Ringvorlesungen zum Thema Rechtsordnung und Juristen im Nationalsozialismus (Gießen, München, Tübingen, Berlin, später in Frankfurt, Kiel und Münster), kirchliche Akademien veranstalteten zahllose entsprechende Wochenenden. Auch die Richterakademien in Trier und Brandenburg nahmen sich schließlich der Thematik an. Das Leitwort war »Vergangenheitsbewältigung ", meist eine solche der $\mathrm{Zi}$ vil- und Strafjustiz, aber auch der Rechtswissenschaft und ihrer, wie man sagte, »Verstrickungen«. Göttingen veranstaltete nun im Wintersemester 2006/07 zum zweiten Mal - nach Ralf Dreier und Wolfgang Sellert (I989) - eine solche Ringvorlesung. Solche Zyklen haben im Laufe der Jahrzehnte ihren Charakter verändert, von muss dazu seine Behauptung, dass mit Worten Recht gemacht wird, in eine andere Richtung gelenkt werden, nämlich in diejenige, dass mit der Wahrnehmung des Genozids als »crime of crimes « eine, wie der Rezensent aus langjähriger Erfahrung in Ruanda weiß, Opferhierarchisierung einhergeht, die der Wiederherstellung des sozialen Friedens in einem Land, das infolge von Bürgerkrieg und Völkermord Opfer auf beiden Seiten kennenlernen musste, überaus abträglich ist. Doch wäre das ein anderes Buch und änderte nichts daran, dass das von Clavero vorgelegte allemal lesenswert ist (was ebenso für die z.T. umfangreichen Anmerkungen gilt), auch wenn man seiner Argumentation nicht immer folgen möchte.

\section{Gerd Hankel}

inhaltlichen und qualitativen Schwankungen abgesehen. Sie können heute mehr voraussetzen als früher, mehr Allgemeinwissen und einen soliden Sockel der Forschung. Insofern müsste vielleicht künftig ein schärfer konturierter Plan zugrunde gelegt werden. Vieles, was sich auch im vorliegenden Sammelband findet, beruht auf Ansätzen der Siebziger- und Achtzigerjahre oder setzt fort, was sich auch anderswo findet. Es wird hier regionalgeschichtlich vertieft (Aufarbeitung der NS-Justiz in Niedersachsen; die Harlan-LüthEntscheidung und ihre Bezüge zu Göttingen), wichtige Figuren werden genauer beleuchtet (Gerhard Leibholz auf der einen, Julius Binder, Karl Larenz und Ernst Rudolf Huber auf der anderen Seite) - oder man findet die alten Themen in erneuerter Gestalt (Rechtswissenschaft

\footnotetext{
* Eva Schumann (Hg.), Kontinuitäten und Zäsuren. Rechtswissenschaft und Justiz im »Dritten Reich « und in der Nachkriegszeit, Göttingen: Wallstein 2008, 375 S., ISBN 978-3-8353-0305-8
} 